\title{
Phylogenetic Classification of Seed Plants of Taiwan
}

\author{
Cheng-Tao Lin ${ }^{1}$ and Kuo-Fang Chung ${ }^{2^{*}}$ (D)
}

\begin{abstract}
Background: Biological classification, the hierarchical arrangement of scientific names of organisms, constitutes the core infrastructure of biological databases. For an efficient management of biological databases, adopting a stable and universal biological classification system is crucial. Currently in Taiwan Biodiversity Information Facility (TaiBIF; http://taibif.tw/), the national portal website that integrates Taiwan's biodiversity information databases, angiosperms are arranged according to Cronquist's System of Classification, which is not compatible with current trend of the Angiosperm Phylogeny Group (APG) classification. To consolidate the function and management of the database, TaiBIF is moving to adopt the APG IV classification and Christenhusz et al. (Phytotaxa 19:55-70, 2011)'s classification of gymnosperms, which we summarize as the Phylogenetic Classification of Seed Plants of Taiwan.
\end{abstract}

Results: The Phylogenetic Classification of Seed Plants of Taiwan places gymnosperms in five families [vs. eight families in the Flora of Taiwan (FOT)] and angiosperms in 210 families (vs. 193 families in FOT). Three FOT gymnosperm families are synonymized in current treatment. Of the 210 APG IV families, familial circumscriptions of 114 families are identical with FOT and 50 families are recircumscription of FOT, with 46 families newly added. Of the 29 FOT families not included in current classification, two families are excluded and 27 families are synonymized.

Conclusions: The adoption of the Phylogenetic Classification of Seed Plants of Taiwan in TaiBIF will provide better service and efficient management of the nation's biodiversity information databases.

Keywords: Angiosperm Phylogeny Group classification, APG IV, Big new biology, Data cleaning, Flowering plants, Gymnosperms, Spermatophytina, TaiBIF, TaiCOL

\section{Background}

Biological classification, the hierarchical arrangement of scientific names of organisms, provides keywords and links to catalogue and organize biological information (Patterson et al. 2014). Biological classification constitutes the core infrastructure of biological databases (Patterson et al. 2010, 2014). Adopting a stable and universal biological classification system not only is crucial for the users but also fundamental for the efficient management of the databases.

TaiBIF (Taiwan Biodiversity Information Facility; http://taibif.tw/) is the national portal website that integrates Taiwan's biodiversity information (Shao et al.

\footnotetext{
*Correspondence: bochung@gate.sinica.edu.tw

${ }^{2}$ Research Museum and Herbarium (HAST), Biodiversity Research Center, Academia Sinica, Taipei 11529, Taiwan

Full list of author information is available at the end of the article
}

2013) through TaiCOL (Catalogue of Life in Taiwan; http://col.taibif.tw/), TaiEOL (Taiwan Encyclopedia of Life; http://eol.taibif.tw/), TaiBOL (Cryobanking Program for Wildlife Genetic Material in Taiwan; http:// cryobank.museum.biodiv.tw/), and TELDAP (Taiwan e-Learning and Digital Archives Programs; http://core. teldap.tw/). As an associate participant of GBIF (Global Biodiversity Information Facility; http://www.gbif.org/), TaiBIF also functions as a national node of GBIF (Shao et al. 2013). The initiation of TaiBIF started in 2003 with the establishment of TaiBNET (Taiwan Biodiversity National Information Network; http://taibnet.sinica. edu.tw), providing "Taiwan species checklist" and the list of local taxonomic experts (Shao et al. 2013). Currently in TaiCOL, the successor of TaiBNET, the flowering plants are arranged according to Cronquist (1968)'s System of Classification (Shao et al. 2008), replacing A. 
Engler's Syllabus der Pflanzenfamilien that was adopted in the Flora of Taiwan (FOT), 2nd edition (Huang 1994). Although Cronquist's System was highly influential and had been followed by several major floras such as Flora of North America (Reveal 1993) and Flora of Australia (Kanis et al. 1999), much of the content of Cronquist System is not compatible to the current trend of the APG classification.

The Angiosperm Phylogeny Group (APG) classification of the orders and families of flowering plants, now in its fourth edition (APG IV), is a collaborative effort of plant molecular systematic community worldwide (The Angiosperm Phylogeny Group 1998, 2003, 2009, 2016), providing the greatest stability and predictability regarding biodiversity information of flowering plants (Mayr 1981; Wearn et al. 2013). Although APG classification has not been adopted officially in Taiwan, families circumscribed by molecular phylogenetic studies and summarized by APG have been increasingly accepted by both academic (Hsu et al. 2011, 2016a, b; Wu et al. 2015) and citizen scientists (e.g., Nature Campus http://nc.biodiv.tw/bbs/ index.php).

As an official provider of biodiversity information of the country, the classification systems followed by TaiCOL has deep and profound influences. In an effort to consolidate the function and management of TaiBIF that shall result in stable and better services of the websites, it is inevitable for TaiCOL to adopt classification systems that are constructed based on results of robust molecular phylogenetic analyses. This article outlines phylogenetic classification of families of the seed plants of Taiwan summarized based on Christenhusz et al. (2011)'s classification of gymnosperms, APG IV, and subsequent studies. To facilitate the transition toward APG IV, we also provide the spreadsheet of the classification schema for all seed plant genera that will be adopted by TaiCOL (Additional file 1: Appendix S1). This spreadsheet will be updated constantly and can be downloaded through TaiCOL. A brief note is provided for families of which circumscription has been changed between the treatment of FOT and APG IV classification.

\section{Methods}

The database of seed plants of Taiwan was compiled from "a checklist of the vascular plants of Taiwan" of the Flora of Taiwan (Boufford et al. 2003), "Illustrated Guide to Aquatic Plants of Taiwan" (Yang et al. 2001), Wu et al. (2010) that summarized naturalized and invasive flora, subsequently published native (e.g., Hsu et al. 2011; Wu et al. 2015) and naturalized (e.g., Liang et al. 2011; Wang et al. 2016) species, and the flora of Tongsha (Pratas) Island (Huang et al. 1994; Lin et al. 2005). The checklist was then imported into relational PostgreSQL database as a basis for migrating process. The migration process applied a 'data cleaning framework' to improve our data set quality through diagnosing, detecting, and correcting procedures. The data cleaning procedure included three major stages: (1) error type defining, (2) error instance identifying, and (3) error correcting (Maletic and Marcus 2000). Furthermore, we followed the data cleaning principles and methods suggested by Chapman (2005) when processing nomenclature data. In the initial stage of migration, instead of constructing a name-based database, a taxon-based database, which includes a unique taxonomy identifier (taxon ID) and several attributes such as family, genus, scientific names and vernacular names, etc., was constructed. In order to reduce the redundancy of the database and improve the data quality and integrity, we adopted relational database normalization to parse the raw data table into a second normal form schema. Through the normalization process, potential errors such as duplicate entries, misplaced taxa, etc., could be eliminated efficiently. In the second stage, we automated a python script to cross-validate our data base with Missouri Botanical Garden's Tropicos (http:// www.tropicos.org/) and International Plant Names Index (IPNI, http://ipni.org), identifying unmatched or unfound names for manual checking. In the third stage, three major possible errors or problems: (1) illegitimate or invalid names, (2) misspelled names, (3) different taxonomic treatment, were corrected after cross-validation.

We adopted Ruggiero et al. (2015) for the higher level classification of seed plants (Subphylum Spermatophytina and above). For gymnosperms (Superclass Gymnospermae), Christenhusz et al. (2011)'s classification was followed, though caution was taken for the uncertainty of the phylogenetic position of gnetophytes ( $\mathrm{Lu}$ et al. 2014; Wang and Ran 2014). For angiosperms (Superclass Angiospermae), major clades recognized as superorders in Chase and Reveal (2009) and the classification of The Angiosperm Phylogeny Group (2016) was adopted, with the exception of Boraginales in which Luebert et al. (2016)'s new familial classification was followed. For orders and families of which vernacular names are lacking in the current literature of the flora of Taiwan, the names proposed by Liu et al. (2015) were followed.

\section{Results and discussion}

Based on Christenhusz et al. (2011), APG IV (2016), and Luebert et al. (2016)'s familial classification of Boraginales, the "Phylogenetic Classification of Seed Plants of Taiwan" is presented below. Of the four classes (I-IV), eight orders (A-H), and 12 families of gymnosperms in Christenhusz et al. (2011)'s classification, five families in four orders of two classes are naturally distributed in Taiwan. Compared to the treatment in FOT, current classification 
includes Taxodiaceae in Cupressaceae and expands Taxaceae to include Amentotaxaceae and Cephalotaxaceae. Of the 16 superorders (A-S), 64 orders, and 427 families currently circumscribed (Luebert et al. 2016; APG IV 2016; Thulin et al. 2016), 210 families in 53 orders of 15 superorders are recorded in the flora of Taiwan (Fig. 1; Table 1).

The adoption of APG IV affects 79 (64 in dicotyledons and 15 in monocotyledons) of the 193 families (152 in dicotyledons and 41 in monocotyledons) recorded in FOT (Boufford et al. 2003), including changes in familial recircumscriptions in 50 families (41 in dicotyledons and 9 in monocotyledons), synonymization of 27 families (21 in dicotyledons and 6 in monocotyledons), and two families not present in Taiwan (i.e., Rafflesiaceae and Hydrophyllaceae). Circumscriptions of 114 (59.1\%) families [88 (57.9\%) in dicotyledons and $26(63.4 \%)$ in monocotyledons] of FOT remain unchanged. A total of 46 families are added under current classification. Table 1 summarizes current status of the families recorded in FOT (Boufford et al. 2003), newly added families (46), and frequently cultivated families not recorded in FOT (20).

The adoption of APG IV inevitably results in changes in statistics of the flora of Taiwan. For examples, Euphorbiaceae s.l. (89 species in 27 genera), Scrophulariaceae s.l. (73 species in 26 genera), and Liliaceae s.l. (48 species in 21 genera) were ranked as the 8th, 10th and 18th most species-rich families in FOT (Hsieh 2003); however, under APG IV classification, Euphorbiaceae reduces to ca. 60 species in 17 genera, Scrophulariaceae to only 4 species in 3 genera, and Liliaceae to ca. 8 species in 2 genera. Saxifragaceae is another case of drastic changes, reducing from 25 species in 13 genera to 7 species in 4 genera. On the other hand, families such as Malvaceae, Plantaginaceae, and Orobanchaceae expand greatly, increasing from eight, one, and four genera to 26,16 , and 14 genera, respectively.

In the Classification outlined below, codes composed of alphabet and number(s) are applied to each family to denote its ordinal (and superordinal) classification. For gymnosperms, Christenhusz et al. (2011)'s alphabetical $(\mathrm{A}-\mathrm{H})$ and numeric (1-12) codes for Orders and Families are adopted. For angiosperms, the numeric codes of APG IV families (1-416) are followed, with the addition of alphabetical (A-S) code for superorders and numerical (1-64) codes for orders modified from Chase and Reveal (2009). For example, “F14.60. Liliaceae 百合科” indicates Superorder Lilianae (F), Order Liliales (14), and Family Liliaceae (60). Code designations of superorders and orders are outlined in Fig. 1. The numeric family codes used in the Flora of Taiwan (Boufford et al. 2003) are also listed in parentheses after the Chinese vernacular family name to aid an easy comparison to families circumscribed in FOT. For examples, "(三IIA.1)" in Cycadaceae indicates Family 1 of Gymnosperma (IIA) in Boufford et al. (2003), “( $\equiv$ IIBa.35)” of Nymphaeaceae denotes Family 35 of Dicotylendons (IIBa), and "(三 IIBb.7)" of Zosteraceae stands for Family 7 of Monocotyledons (IIBb), while the sign " $\equiv$ " indicates unchanged familial circumscription between FOT and current treatment.

For those families of which circumscription has been changed, the number of genera in FOT and current treatment are also provided. For examples, "F14.60. Liliaceae 百合科 (IIBb.9; 21/2)" indicates 21 genera included in Family 9 of Monocotyledons (IIBb) in FOT, while only 2 genera are included in current classification. A statement is followed to denote newly added genera and/or genera excluded. For examples, current classification of “F18.89. Zingiberaceae 薑科 (IIBb.35; 5/5)" includes the genus Curcuma 薑黃屬 based on Wu et al. (2010), while the genus Costus of FOT is moved to F18.88 Costaceae, resulting in a total of five genera as recorded in FOT (5/5). For newly added families, genera included are listed with references to previous classification. For newly recorded families, the number in parentheses after the Chinese vernacular name indicate the number of genera included. For families whose circumscription remain unchanged (e.g., Lauraceae, Asteraceae, Fabaceae, Orchidaceae, etc.), the full list of genera included is summarized in Additional file 1: Appendix S1. The Chinese vernacular names for all scientific names of taxa are adopted from FOT and/or names proposed when published (e.g., Chung et al. 2010b; Hsu et al. 2011). For newly added taxa not published by Taiwanese authors, the Chinese names proposed by Liu et al. (2015) are adopted. Although not officially recorded as parts of the flora, current treatments of 21 frequently cultivated plant families in Taiwan not recorded in FOT (marked with *) are also included.

\section{Conclusions}

Phylogenetic Classification of Seed Plants of Taiwan

Kingdom Plantae 植物界, Subkingdom Viridiplantae 綠 色植物亞界, Infrakingdom Streptophyta 鏈型植物次界, Superphylum Embryophyta 有胚植物超門, Phylum Tracheophyta 維管束植物門, Subphylum Spermatophytina 種子植物亞門 (Ruggiero et al. 2015)

Superclass Gymnospermae 裸子植物超綱 (三 gymnosperms in Chase and Reveal 2009 and Christenhusz et al. 2011)

Class I. Cycadopsida 蘇鐵綱, Subclass Cycadidae 蘇鐵 亞綱

A. Cycadales 蘇鐵目

A.1. Cycadaceae 蘇鐵科 (三IIA.1)

"A.2. Zamiaceae 藏米亞 (堅果鳳尾蕉) 科 


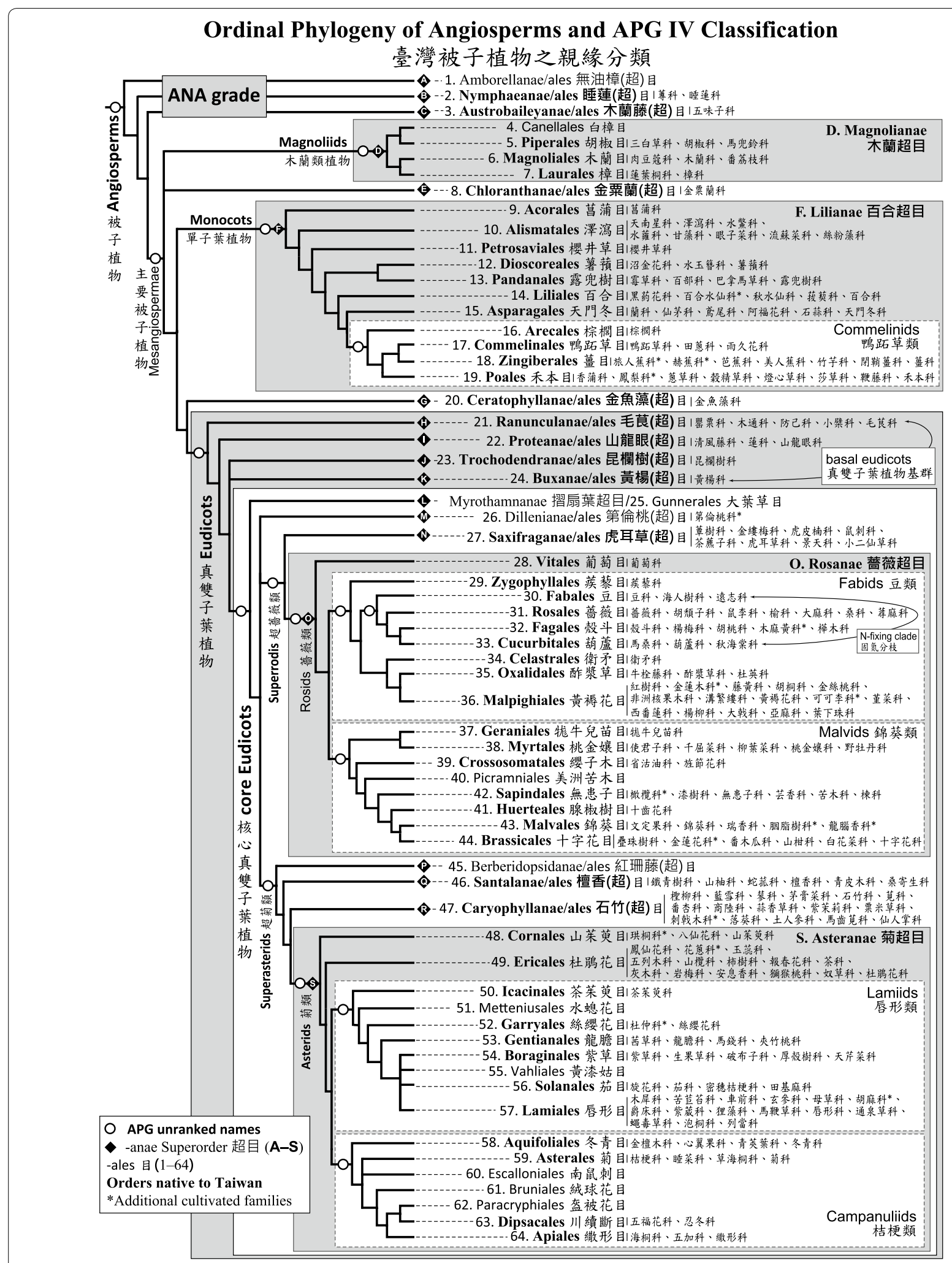

Fig. 1 Ordinal phylogeny of angiosperms and APG IV classification, with notes on familial classification of angiosperm families of Taiwan 
Table 1 Current taxonomic status of the families recorded in the Flora of Taiwan (FOT), 2nd ed. (Boufford et al. 2003) and newly added families to the flora of Taiwan based on APG IV

FOT families excluded (2)
FOT families synonymized (27)

FOT families circumscription changed (50)

FOT families circumscription unchanged (114)
IIBa.43 Rafflesiaceae and IIBa.125 Hydrophyllaceae

IIBa.7A Cecropiaceae, IIBa.20 Chenopodiaceae, IIBa.26 IIliciaceae, IIBa.48A Fumariaceae, IIBa.70 Aceraceae, IIBa.71A Bretschneideraceae, IIBa.81 Leeaceae, IIBa.83 Tiliaceae, IIBa.85 Bombacaceae, IIBa.86 Sterculiaceae, IIBa.89 Flacourtiaceae, IIBa.101A Trapaceae, IIBa.103 Theligonaceae, IIBa.104 Alangiaceae, IIBa.109 Pyrolaceae, IIBa.111 Myrsinaceae, IIBa.122 Asclepiadaceae, IIBa.128 Callitrichaceae, IIBa.137 Myoporaceae, IIBa.140 Valerianaceae, IIBa.141 Dipsacaceae, IIBb.6 Zannichelliaceae, IIBb.8 Najadaceae, IIBb.11 Agavaceae, IIBb.28(A) Taccaceae, IIBb.30 Lemnaceae, and IIBb.33 Sparganiaceae

IIBa.3 Salicaceae, IIBa.6 UImaceae, IIBa.7 Moraceae, IIBa.8 Urticaceae, IIBa.9A Olacaceae, IIBa.10 Santalaceae, IIBa.11 Loranthaceae, IIBa.14 Phytolaccaceae, IIBa.18 Portulacaceae, IIBa.21 Amaranthaceae, IIBa.25 Schisandraceae, IIBa.45 Theaceae, IIBa.46 Clusiaceae (三 Guttiferae), IIBa.48 Papaveraceae, IIBa.49 Capparaceae, IIBa.51 Hamamelidaceae, IIBa.53 Saxifragaceae, IIBa.61 Euphorbiaceae, IIBa.71 Sapindaceae, IIBa.75 Celastraceae, IIBa.78 Icacinaceae, IIBa.80 Vitaceae, IIBa.84 Malvaceae, IIBa.95 Lythraceae, IIBa.105 Cornaceae, IIBa.106 Araliaceae, IIBa.107 Apiaceae (三 Umbelliferae), IIBa.110 Ericaceae, IIBa.112 Primulaceae, IIBa.119 Loganiaceae, IIBa.120 Gentianaceae, IIBa.121 Apocynaceae, IIBa.123 Rubiaceae, IIBa.126 Boraginaceae, IIBa.127 Verbenaceae, IIBa.129 Lamiaceae (三 Labiatae), IIBa.131 Scrophulariaceae, IIBa.133 Acanthaceae, IIBa.135 Orobanchaceae, IIBa.138 Plantaginaceae, IIBa.139 Caprifoliaceae, IIBb.1 Alismataceae, IIBb.2 Hydrocharitaceae, IIBb.4 Potamogetonaceae, IIBb.9 Liliaceae, IIBb.12 Amaryllidaceae, IIBb.14 Dioscoreaceae, IIBb.29 Araceae, IIBb.33 Typhaceae, and IIBb.35 Zingiberaceae

IIBa.1 Myricaceae, IIBa.2 Juglandaceae, IIBa.4 Betulaceae, IIBa.5 Fagaceae, IIBa.9 Proteaceae, IIBa.10A, Opiliaceae, IIBa.12 Balanophoraceae, IIBa.13 Polygonaceae, IIBa.15 Nyctaginaceae, IIBa.16 Molluginaceae, IIBa.17 Aizoaceae, IIBa.18A Basellaceae, IIBa.19 Caryophyllaceae, IIBa.22 Magnoliaceae, IIBa.23 Annonaceae, IIBa.24 Myristicaceae, IIBa.27 Lauraceae, IIBa.28 Hernandiaceae, IIBa.29 Trochodendraceae, IIBa.30 Ranunculaceae, IIBa.31 Berberidaceae, IIBa.32 Lardizabalaceae, IIBa.33 Menispermaceae, IIBa.34 Nelumbonaceae, IIBa.35 Nymphaeaceae, IIBa.36 Cabombaceae, IIBa.38 Ceratophyllaceae, IIBa.39 Saururaceae, IIBa.40 Piperaceae, IIBa.41 Chloranthaceae, IIBa.42 Aristolochiaceae, IIBa.44 Actinidiaceae, IIBa.47 Droseraceae, IIBa.50 Brassicaceae (三 Cruciferae), IIBa.52 Crassulaceae, IIBa.54 Pittosporaceae, IIBa.55 Rosaceae, IIBa.56 Connaraceae, IIBa.57 Fabaceae (三 Leguminosae), IIBa.58 Oxalidaceae, IIBa.59 Geraniaceae, IIBa.60 Zygophyllaceae, IIBa.62 Daphniphyllaceae, IIBa.63 Rutaceae, IIBa.64 Simaroubaceae, IIBa.65 Meliaceae, IIBa.66 Malpighiaceae, IIBa.67 Polygalaceae, IIBa.68 Coriariaceae, IIBa.69 Anacardiaceae, IIBa.72 Sabiaceae, IIBa.73 Balsaminaceae, IIBa.74 Aquifoliaceae, IIBa.76 Staphyleaceae, IIBa.77 Buxaceae, IIBa.79 Rhamnaceae, IIBa.82 Elaeocarpaceae, IIBa.87 Thymelaeaceae, IIBa.88 Elaeagnaceae, IIBa.90 Violaceae, IIBa.91 Stachyuraceae, IIBa.91 A Passifloraceae, IIBa.92 Elatinaceae, IIBa.93 Begoniaceae, IIBa.94 Cucurbitaceae, IIBa.96 Myrtaceae, IIBa.97 Lecythidaceae, IIBa.98 Melastomataceae, IIBa.99 Rhizophoraceae, IIBa.100 Combretaceae, IIBa.101 Onagraceae, IIBa.102 Haloragaceae, IIBa.108 Diapensiaceae, IIBa.113 Plumbaginaceae, IIBa.114 Sapotaceae, IIBa.115 Ebenaceae, IIBa.116 Styracaceae, IIBa.117 Symplocaceae, IIBa.118 Oleaceae, IIBa.124 Convolvulaceae, IIBa.130 Solanaceae, IIBa.132 Bignoniaceae, IIBa.134 Gesneriaceae, IIBa.136 Lentibulariaceae, IIBa.142 Campanulaceae, IIBa.142A Sphenocleaceae, IIBa.143 Goodeniaceae, IIBa.144 Asteraceae (三 Compositae), IIBb.3 Aponogetonaceae, IIBb.5 Ruppiaceae, IIBb.7 Zosteraceae, IIBb.9A Petrosaviaceae, IIBb.10 Stemonaceae, IIBb.13 Hypoxidaceae, IIBb.15 Smilacaceae, IIBb.16 Pontederiaceae, IIBb.17 Iridaceae, IIBb.18 Burmanniaceae, IIBb.19 Philydraceae, IIBb.20 Juncaceae, IIBb.21 Commelinaceae, IIBb.22 Xyridaceae, IIBb.23 Eriocaulaceae, IIBb.24 Flagellariaceae, IIBb.25 Cyperaceae, IIBb.26 Poaceae (三 Gramineae), IIBb.27 Arecaceae (三 Palmae), IIBb.28 Cyclanthaceae, IIBb.31 Pandanaceae, IIBb.34 Musaceae, IIBb.36 Cannaceae, IIBb.37 Marantaceae, IIBb.38 Orchidaceae, and IIBb.39 Triuridaceae

F9.27 Acoraceae, F10.41 Cymodoceaceae, F12.43 Nartheciaceae, F14.53 Melanthiaceae, F14.56 Colchicaceae, F15.72 Asphodelaceae, F15.74 Asparagaceae, F18.88 Costaceae, N27.123 Altingiaceae, N27.127 Iteaceae, N27.128 Grossulariaceae, O30.141 Surianaceae, O31.149 Cannabaceae, O36.184 Calophyllaceae, and O36.186 Hypericaceae, O36.189 Putranjivaceae, O36.208 Linaceae, O36.211 Phyllanthaceae, O41.234 Dipentodontaceae, O43.245 Muntingiaceae, O44.254 Akaniaceae, O44.257 Caricaceae, O44.269 Cleomaceae, Q46.278 Schoepfiaceae, R47.281 Tamaricaceae, R47.306 Petiveriaceae, R47.314 Talinaceae, R47.317 Cactaceae, S48.320 Hydrangeaceae, S49.332 Pentaphylacaceae, S49.345 Mitrastemonaceae, S52.351 Garryaceae, S54.357B Coldeniaceae, S54.357C Cordiaceae, S54.357D Ehretiaceae, S54.357E Heliotropiaceae, S56.363 Hydroleaceae, S57.373 Linderniaceae, S57.384 Mazaceae, S57.385 Phrymaceae, S57.386 Paulowniaceae, S58.388 Stemonuraceae, S58.389 Cardiopteridaceae, S58.391 Helwingiaceae, S59.400 Menyanthaceae, and S63.408 Adoxaceae

A.2 Zamiaceae, B.3 Ginkgoaceae, G.8 Araucariaceae, F14.55 Alstroemeriaceae, F18.82 Strelitziaceae, F18.84 Heliconiaceae, F19.91 Bromeliaceae, M26.120 Dilleniaceae, O32.156 Casuarinaceae, O36.181 Ochnaceae, O36.197 Chrysobalanaceae, O42.238 Burseraceae, O43.250 Bixaceae, O43.253 Dipterocarpacea, O44.255 Tropaeolaceae, R47.311 Didiereaceae, S48.318 Nyssaceae, S49.329 Polemoniaceae, S52.350 Eucommiaceae, and S57.376 Pedaliaceae 
Class II. Ginkgoopsida 銀杏綱, Subclass Ginkgooidae 銀杏亞綱

B. Ginkgoales 銀杏目

"B.3. Ginkgoaceae 銀杏科

Class III. Gnetopsida 買麻藤綱, Subclass Gnetidae 買 麻藤亞綱
C. Welwitschiales 二葉樹目
D. Gnetales 買麻藤目
E. Ephedrales 麻黃目

Class IV. Pinopsida 松綱, Subclass Pinidae 松亞綱

F. Pinales 松目

F.7. Pinaceae 松科 (三IIA.6)

G. Araucariales 南洋杉目

"G.8. Araucariaceae 南洋杉科

G.9. Podocarpaceae 羅漢松科 (三 IIA.5)

H. Cupressales 柏目

H.11. Cupressaceae 柏科 (IIA.8; 3/5)

Including IIA.7 Taxodiaceae (Cryptomeria 柳 杉屬, Cunninghamia 杉木屬 and Taiwania 臺 灣杉屬).

H.12. Taxaceae 紅豆杉科 (IIA.2; 1/3)

Including IIA.3 Amentotaxaceae (Amentotaxus 穗花杉屬) and IIA.4 Cephalotaxaceae (Cephalotaxus 粗榧屬).

Superclass “Angiospermae" 被子植物超綱, Class Magnoliopsida 木蘭植物綱, Subclass Magnoliidae 木蘭植物 亞綱 (三 angiosperms in Chase and Reveal 2009; APG IV 2016)

Amborellanae 無油樟超目

A1. Amborellales 無油樟目

B. Nymphaeanae 睡蓮超目

B2. Nymphaeales 睡蓮目

B2.3. Cabombaceae 蓴科 (三IIBa.36; 1/2)

Adding Cabomba 穗專屬 (Yang et al. 2001).

B2.4. Nymphaeaceae 睡蓮科 (三IIBa.35)

C. Austrobaileyanae 木蘭藤 (昆士蘭樟) 超目

C3. Austrobaileyales 木蘭藤 (昆士蘭樟) 目

C3.7. Schisandraceae 五味子科 (IIBa.25; 2/3)

Including IIBa.26 Illiciaceae (Illicium八角屬).

D. Magnolianae 木蘭超目

D4. Canellales 白樟 (白桂皮) 目

D5. Piperales 胡椒目

D5.10. Saururaceae 三白草科 (三IIBa.39)

D5.11. Piperaceae 胡椒科 (三 IIBa.40)

D5.12. Aristolochiaceae 馬㝸鈴科 (三IIBa.42)
D6. Magnoliales 木蘭目

D6.13. Myristicaceae 肉荳冦科 (三IIBa.24)

D6.14. Magnoliaceae 木蘭科 (三IIBa.22)

D6.18. Annonaceae 番荔枝科 (三IIBa.23)

D7. Laurales 樟目

D7.23. Hernandiaceae 蓮葉桐科 (三IIBa.28)

D7.25. Lauraceae 樟科 (三IIBa.27)

E. Chloranthanae 金粟蘭超目

E8. Chloranthales 金粟蘭目

E8.26. Chloranthaceae 金粟蘭科 (三IIBa.41)

F. Lilianae 百合超目 (Trias-Blasi et al. 2015)

F9. Acorales 菖蒲目

F9.27. Acoraceae 菖蒲科 (1)

Acorus 菖蒲屬 (IIBb.29 Araceae).

F10. Alismatales 澤瀉目

F10.28. Araceae 天南星科 (IIBb.29; 16/20)

Adding Syngonium 合果芋屬 (Wu et al. 2010);

excluding F9.27 Acoraceae (Acorus); including

IIBb.30 Lemnaceae [Landoltia ( $\equiv$ Spirodela punctata) 蘭氏萍屬 (Les and Crawford 1999), Lemna 青萍屬, Spirodela 浮萍屬, and Wolffia 無根萍屬).

F10.30. Alismataceae 澤瀉科 (IIBb.1; 3/5)

Including Limnocharitaceae (Hydrocleys 水罌 粟屬 and Limnocharis 黃花藺屬; Yang et al. 2001).

F10.32. Hydrocharitaceae 水鱉科 (IIBb.2; 7/10) Adding Egeria 水蘊草屬 (Wu et al. 2010) and Limnobium 南美海綿屬 (Wu et al. 2010); incluidng IIBb.8 Najadaceae (Najas 拂尾藻屬).

F10.34. Aponogetonaceae 水䔨科 (三IIBb.3)

F10.37. Zosteraceae 甘藻科 (三 IIBb.7)

F10.38. Potamogetonaceae 眼子菜科 (IIBb.4; 1/3) Adding Stuckenia (三 Potamogeton pectinatus) 篦齒眼子菜屬 (Lindqvist et al. 2006); including IIBb.6 Zannichelliaceae pro parte (Zannichellia 角果藻屬)。

F10.40. Ruppiaceae 流蘇菜科 (三IIBb.5)

F10.41. Cymodoceaceae 絲粉藻科 (3)

IIBb.4 Potamogetonaceae pro parte (Cymodocea 絲粉藻屬 and Syringodium 針葉藻屬; Lin et al. 2005) and IIBb.6 Zannichelliaceae pro parte (Halodule 二葯藻屬). Based on Ko (2004), the photographs of Lin et al. (2005; Fig. 5a, b) identified as Thalassodendron ciliatum are likely misidentification of Cymodocea serrulata. 
F11. Petrosaviales 櫻井草 (無葉蓮) 目 F11.42. Petrosaviaceae 櫻井草 (無葉蓮) 科 (三 IIBb.9A)

F12. Dioscoreales 薯蕷目

F12.43. Nartheciaceae 沼金花 (納茜菜) 科 (1) Aletris 粉條兒屬 (IIBb.9 Liliaceae)

F12.44. Burmanniaceae 水玉䙃科 (三IIBb.18)

F12.45. Dioscoreaceae 薯蕷科 (IIBb.14; 1/2) Including IIBb.28(A) Taccaceae (Tacca 蒟蒻薯 屬).

F13. Pandanales 露㝸樹目

F13.46. Triuridaceae 霉草科 (三IIBb.39)

F13.48. Stemonaceae 百部科 (三IIBb.10)

F13.49. Cyclanthaceae 巴拿馬草科 (三IIBb.28)

F13.50. Pandanaceae 露㝸樹科 (三IIBb.31)

F14. Liliales 百合目

F14.53. Melanthiaceae 黑药花科 (5) Ypsilandra ( $($ 芯花屬; Hsu et al. 2011) and IIBb.9 Liliaceae pro. parte (Helonias 胡麻花屬, Paris 七葉一枝花屬, Trillium 延齡草屬, and Veratrum 藜蘆屬).

"F14.55. Alstroemeriaceae 百合水仙 (六出花) 科

F14.56. Colchicaceae 秋水仙科 (1) Disporum 寶鐸花屬 (IIBb.9 Liliaceae).

F14.59. Smilacaceae 菝荕科 (三IIBb.15)

F14.60. Liliaceae 百合科 (IIBb.9; 21/2) Lilium (百合屬) and Tricyrtis (油點草屬); excluding F14.53 Melanthiaceae (Helonias, Paris, Trillium, and Veratrum), F14.56 Colchicaceae (Disporum), F15.72 Asphodelaceae (Dianella and Hemerocallis), F15.73 Amaryllidaceae pro parte (Allium), and F15.74 Asparagaceae pro parte [Asparagus, Aspidistra, Disporopsis, Liriope, Ophiopogon, Peliosanthes, Polygonatum, Rohdea (三Campylandra), Scilla (三Barnardia), Smilacina (三 Maianthemum), and Thysanotus].

F15. Asparagales 天門冬目

F15.61. Orchidaceae 蘭科 (三IIBb.38)

F15.66. Hypoxidaceae 仙茅科 (三IIBb.13)

F15.70. Iridaceae 蔦尾科 (三IIBb.17)

F15.72. Asphodelaceae 阿福花 (獨尾草) 科 (2) IIBb.9 Liliaceae pro parte (Dianella 桔梗蘭屬 and Hemerocallis 萱草屬).

F15.73. Amaryllidaceae 石蒜科 (IIBb.12; 2/3) Including Allium 葱屬 (IIBb.9 Liliaceae).

F15.74. Asparagaceae 天門冬科 (16) Including IIBb.11 Agavaceae (Agave 龍舌蘭 屬, Cordyline 朱蕉屬, Dracaena 龍血樹屬, and Yucca 金棒蘭屬) and IIBb.9 Liliaceae pro. parte [Asparagus 天門冬屬, Aspidistra 蜘蛛抱 蛋屬, Barnardia (三Scilla sinensis) 綿霜兒屬, Disporopsis 假寶鐸花屬, Heteropolygonatum 異黃精屬 (Chao et al. 2013), Liriope 麥門冬屬, Maianthemum 鹿藥屬 (舞鶴草) 屬, Ophiopogon 沿階草屬, Peliosanthes 球子草屬, Polygonatum 黃精屬, Rohdea (三Campylandra) 萬 年青屬 (Yamashita and Tamura 2004), and Thysanotus 異荵草屬].

F16. Arecales 棕㯗目

F16.76. Arecaceae (三 Palmae) 棕㯗科 (三IIBb.27)

F17. Commelinales 鴨跖草目

F17.78. Commelinaceae 鴨跖草科 (三IIBb.21)

F17.79. Philydraceae 田葸科 (三IIBb.19)

F17.80. Pontederiaceae 雨久花科 (三IIBb.16)

F18. Zingiberales 薑目

"F18.82. Strelitziaceae 旅人蕉科

"F18.84. Heliconiaceae 蠍尾蕉 (赫蕉) 科

F18.85. Musaceae 芭蕉科 (三IIBb.34)

F18.86. Cannaceae 美人蕉科 (三IIBb.36)

F18.87. Marantaceae 竹芋科 (三IIBb.37)

F18.88. Costaceae 閉鞘薑科 (1)

Costus 閉鞘薑屬 (IIBb.35 Zingiberaceae).

F18.89. Zingiberaceae 薑科 (IIBb.35; 5/5)

Adding Curcuma 畐黃屬 (Wu et al. 2010); excluding F18.88. Costaceae (Costus).

F19. Poales 禾本目

F19.90. Typhaceae 香蒲科 (IIBb.33; 1/2)

Including IIBb.33 Sparganiaceae (Sparganium 黑三稜屬).

"F19.91. Bromeliaceae 鳳梨科

F19.93. Xyridaceae 葸草科 (三IIBb.22)

F19.94. Eriocaulaceae 穀精草科 (三IIBb.23)

F19.97. Juncaceae 燈心草科 (三IIBb.20)

F19.98. Cyperaceae 莎草科 (三IIBb.25)

F19.100. Flagellariaceae 鞭藤科 (三IIBb.24)

F19.103. Poaceae (三 Gramineae) 禾本科 (三 IIBb.26)

G. Ceratophyllanae 金魚藻超目

G20. Ceratophyllales 金魚藻目

G20.104. Ceratophyllaceae 金魚藻科 (三IIBa.38)

H. Ranunculanae 毛茛超目

H21. Ranunculales 毛莨目

H21.106. Papaveraceae 罌粟科 (IIBa.48; 3/5) Including IIBa.48A Fumariaceae (Corydalis 紫 蓳屬 and Fumaria 球果紫堇屬). 
H21.108. Lardizabalaceae 木通科 (三IIBa.32)

H21.109. Menispermaceae 防己科 (三IIBa.33)

H21.110. Berberidaceae 小檗科 (三IIBa.31)

H21.111. Ranunculaceae 毛茛科 (三IIBa.30)

I. Proteanae 山龍眼超目

I22. Proteales 山龍眼目

I22.112. Sabiaceae 清風藤科 (三IIBa.72)

I22.113. Nelumbonaceae 蓮科 (三 IIBa.34)

I22.115. Proteaceae 山龍眼科 ( $\equiv$ IIBa.9)

J. Trochodendranae 昆欄樹超目

J23. Trochodendrales 昆欄樹目

J23.116. Trochodendraceae 昆欄樹科 (三 IIBa.29)

K. Buxanae 黃楊超目

K24. Buxales 黃楊目

K24.117. Buxaceae 黃楊科 (三 IIBa.77)

L. Myrothamnanae 摺扇葉超目

L25. Gunnerales 大葉草 (洋二仙草) 目

M. Dillenianae 第倫桃超目

M26. Dilleniales 第倫桃目

"M26.120. Dilleniaceae 第倫桃科

N. Saxifraganae 虎耳草超目

N27. Saxifragales 虎耳草目

N27.123. Altingiaceae 草樹 (楓香) 科 (1)

Liquidambar 楓香屬 (IIBa.51 Hamamelidaceae).

N27.124. Hamamelidaceae 金縷梅科 (IIBa.51;

6/5)

Excluding Liquidambar (N27.123).

N27.126. Daphniphyllaceae 虎皮楠科 (三 IIBa.62)

N27.127. Iteaceae 鼠刺科 (1)

Itea 鼠刺屬 (IIBa.53 Saxifragaceae).

N27.128. Grossulariaceae 茶蔍子科 (1)

Ribes 茶蔍子屬 (IIBa.53 Saxifragaceae).

N27.129. Saxifragaceae 虎耳草科 (IIBa.53; 13/5)

Astilbe 落新婦屬, Chrysosplenium 貓兒眼

睛草屬, Mitella 嗩呐草屬, Saxifraga 虎耳草

屬, and Tiarella 黃水枝屬; excluding S48.320

Hydrangeaceae (Cardiandra, Deutzia, Hydran-

gea, Pileostegia, and Schizophragma), N27.127

Iteaceae (Itea), N27.128 Grossulariaceae

(Ribes), and Parnassia (O34.168).

N27.130. Crassulaceae 景天科 (三IIBa.52)

N27.134. Haloragaceae 小二仙草科 (三IIBa.102)

O. Rosanae 薔薇超目

O28. Vitales 葡萄目
O28.136. Vitaceae 葡萄科 (IIBa.80; 6/7)

Including IIBa.81 Leeaceae (Leea 火筒樹屬).

O29. Zygophyllales 蔜藜目

O29.138. Zygophyllaceae 教藜科 (三IIBa.60)

O30. Fabales 豆目

O30.140. Fabaceae (三Leguminosae) 豆科 (三IIBa.57)

O30.141. Surianaceae 海人樹科 (1)

Suriana 海人樹屬 (Huang et al. 1994).

O30.142. Polygalaceae 遠志科 (三IIBa.67)

O31. Rosales 蔷薇目

O31.143. Rosaceae 蔷薇科 (三 IIBa.55)

O31.146. Elaeagnaceae 胡頽子科 (三IIBa.88)

O31.147. Rhamnaceae 鼠李科 (三IIBa.79)

O31.148. Ulmaceae 榆科 (IIBa.6; 5/2)

Ulmus 榆屬 and Zelkova 櫸屬; excluding O31.149 Cannabaceae pro parte (Aphananthe, Celtis, and Trema).

O31.149. Cannabaceae 大麻科 (4)

Humulus 葎草屬 (IIBa.7 Moraceae) and IIBa.6 Ulmaceae pro parte (Aphananthe 粘葉樹屬, Celtis 朴屬, and Trema 山黃麻屬).

O31.150. Moraceae 桑科 (IIBa.7; 8/7)

Excluding Hummulus (O31.149).

O31.151. Urticaceae 僖麻科 (IIBa.8; 21/22) Including IIBa.7A Cecropiaceae (Poikilospermum 錐頭麻屬).

O32. Fagales 殼斗目

O32.153. Fagaceae 殸斗科 ( $\equiv \mathrm{IIBa} .5$ )

O32.154. Myricaceae 楊梅科 (三IIBa.1)

Morella (= Myrica) 楊梅屬 (Herbert 2005;

Huguet et al. 2005).

O32.155. Juglandaceae 胡桃科 (三 IIBa.2)

"O32.156. Casuarinaceae 木麻黃科

O32.158. Betulaceae 樺木科 (三IIBa.4)

O33. Cucurbitales 葫蘆 (瓜) 目

O33.162. Coriariaceae 馬桑科 ( $\equiv \mathrm{IIBa} .68)$

O33.163. Cucurbitaceae 葫蘆 (瓜) 科 (三IIBa.94)

O33.166. Begoniaceae 秋海棠科 (三IIBa.93)

O34. Celastrales 衛矛目

O34.168. Celastraceae 衛矛科 (IIBa.75; 6/6) Including Parnassia 梅花草屬 (IIBa.53 Saxifragaceae); excluding Perrottetia (O41.234).

O35. Oxalidales 酢漿草目

O35.170. Connaraceae 牛栓藤科 (三IIBa.56)

O35.171. Oxalidaceae 酢漿草科 (三IIBa.58)

O35.173. Elaeocarpaceae 杜英科 (三 IIBa.82) 
O36. Malpighiales 黃裖花 (金虎尾) 目 O36.179. Rhizophoraceae 紅樹科 (三IIBa.99)

"O36.181. Ochnaceae 金蓮木科 O36.183. Clusiaceae (三 Guttiferae) 藤黃科 (IIBa.46; 4/1)

Garcinia 福木屬; excluding O36.184 Calophyllaceae (Calophyllum) and O36.186 Hypericaceae (Hypericum and Triadenum).

O36.184. Calophyllaceae 胡桐 (紅厚殼) 科 (1) Calophyllum 胡桐屬 (IIBa.46 Guttiferae).

O36.186. Hypericaceae 金絲桃科 (2) IIBa.46 Guttiferae pro parte (Hypericum 金絲 桃屬 and Triadenum三腺金絲桃屬).

O36.189. Putranjivaceae 非洲核果木 (核果木) 科 (1)

Drypetes (including Liodendron) 鐵色屬 (IIBa.61 Euphorbiaceae).

O36.191. Elatinaceae 溝繁縷科 (三IIBa.92)

O36.192. Malpighiaceae 黃裖花 (金虎尾) 科 (三 IIBa.66)

${ }^{*}$ O36.197. Chrysobalanaceae 可可李 (金殼果) 科 O36.200. Violaceae 堇菜科 (三IIBa.90)

O36.202. Passifloraceae 西番蓮科 (三IIBa.91A)

O36.204. Salicaceae 楊柳科 (IIBa.3) (1/7)

Salix 柳屬; including IIBa.89 Flacourtiaceae (Casearia 嘉賜木屬, Flacourtia 羅庚果屬, Homalium 天料木屬, Idesia 山桐子屬, Scolopia 魯花樹屬, and Xylosma 柞木屬).

O36.207. Euphorbiaceae 大戟科 (IIBa.61; 27/17) Acalypha 鐵莫屬, Alchornea 山麻桿屬, Claoxylon 假鐵莫屬, Croton 巴豆屬, Euphorbia (including Chamaesyce) 大戟屬, Excoecaria 土沉香屬, Homonoia 水楊梅屬, Macaranga 血桐屬, Mallotus 野桐屬, Melanolepis 蟲屎屬, Mercurialis 山靛屬, Homalanthus (三 Omalanthus) 圓葉血桐屬, Ricinus 蓖 麻屬, and Sapium 烏柏屬, and Suregada (including Gelonium) 白樹屬; adding Manihot 木薯屬 and Vernicia (including Aleurites montana; Wu et al. 2010) 油桐屬; excluding O36.189 Putranjivaceae (Drypetes and Liodendron) and O36.211 Phyllanthaceae (Antidesma, Bischofia, Breynia, Bridelia, Flueggea, Glochidion, Margaritaria, Phyllanthus, and Synostemon).

O36.208. Linaceae 亞麻科 (1) Linum 亞麻屬 (Chao et al. 2017).

O36.211. Phyllanthaceae 葉下珠科 (8)

IIBa.61 Euphorbiaceae pro parte (Antidesma 五月茶屬, Bischofia 重陽木屬, Breynia (including Synostemon) 山漆茎屬, Bridelia 土 密樹屬, Flueggea 白飯樹屬, Glochidion 饅頭
果屬, Margaritaria 紫黃屬, and Phyllanthus 油柑屬).

O37. Geraniales 陇牛兒苗目

O37.212. Geraniaceae 陇牛兒苗科 (三IIBa.59)

O38. Myrtales 桃金孃目

O38.214. Combretaceae 使君子科 ( $\equiv \mathrm{IIBa} .100)$

O38.215. Lythraceae 千屈菜科 (IIBa.95; 5/6)

Including IIBa.101A Trapaceae (Trapa 菱屬) and "Punicaceae.

O38.216. Onagraceae 柳葉菜科 (三IIBa.101)

O38.218. Myrtaceae 桃金孃科 (三 IIBa.96)

O38.219. Melastomataceae 野牡丹科 (三 IIBa.98)

O39. Crossosomatales 流蘇子 (縜子木) 目

O39.226. Staphyleaceae 省沽油科 (三 IIBa.76)

O39.228. Stachyuraceae 旌節花科 (三IIBa.91)

O40. Picramniales 美洲苦木目

O41. Huerteales 腺椒樹 (十齒花) 目

O41.234. Dipentodontaceae 十齒花科 (1) Perrottetia 核子小屬 (IIBa.75 Celastraceae).

O42. Sapindales 無患子目

"O42.238. Burseraceae 橄欖科

O42.239. Anacardiaceae 漆樹科 ( IIBa.69)

O42.240. Sapindaceae 無患子科 (IIBa.71; 9/10)

Including IIBa.70 Aceraceae (Acer 楓屬).

O42.241. Rutaceae 芸香科 ( $\equiv$ IIBa.63)

O42.242. Simaroubaceae 苦木科 (三IIBa.64)

O42.243. Meliaceae 楝科 (三IIBa.65)

O43. Malvales 錦葵目

O43.245. Muntingiaceae 文定果 (西印度櫻桃) 科 (1)

Muntingia 西印度櫻桃屬 (IIBa.83 Tiliaceae).

O43.247. Malvaceae 錦葵科 (IIBa.84; 8/26)

Abelmoschus 秋葵屬, Abutilon 茼麻屬, Hibiscus 木槿屬, Malachra 玄葵屬, Malva 錦葵 屬, Malvastrum 賽葵屬, Sida 金午時花屬, Thespesia 繖楊屬, and Urena 野棉花屬; adding Anoda 蔓錦葵屬 (Li and Wang 2012) and Modiola 蒀葵屬 (Wu et al. 2010); including IIBa.85 Bombacaceae (Bombax 木棉屬 and Pachira 馬拉巴栗屬; Wu et al. 2010), IIBa.86 Sterculiaceae (Firmiana 梧桐屬, Helicteres 山 芝麻屬, Heritiera 銀葉樹屬, Kleinhovia 克蘭 樹屬, Melochia 野路葵屬, Pterospermum 翅 子樹屬, Reevesia 梭羅樹屬, Sterculia 蘋婆屬, and Waltheria 草梧桐屬) and IIBa.83 Tiliaceae pro parte (Berrya 六翅木屬, Corchorus 黃麻 
屬, Grewia 捕魚木屬, and Triumfetta 垂桉草 屬).

O43.249. Thymelaeaceae 瑞香科 (三IIBa.87)

${ }^{*}$ O43.250. Bixaceae 胭脂樹 (紅木) 科

*O43.253. Dipterocarpaceae 龍腦香科

O44. Brassicales 十字花目

O44.254. Akaniaceae 疊珠樹科 (1) Including IIBa.71A Bretschneideraceae (Bretschneidera 鐘葶木屬).

*O44.255. Tropaeolaceae 金蓮花 (旱金蓮) 科

O44.257. Caricaceae 番木瓜科 (1)

Carica 番木瓜屬 (Wu et al. 2010).

O44.268. Capparaceae 山柑科 (IIBa.49; 3/2)

Excluding Cleome (O44.269).

O44.269. Cleomaceae 白花菜 (醉蝶花) 科 (1) Cleome 白花菜屬 (IIBa.49 Capparaceae).

O44.270. Brassicaceae (三 Cruciferae) 十字花科 ( $\equiv$ IIBa.50)

P. Berberidopsidanae 紅珊藤 (智利藤) 超目

P45. Berberidopsidales 紅珊藤 (智利藤) 目

Q. Santalanae 檀香超目

Q46. Santalales 檀香目

Q46.273. Olacaceae 鐵青樹科 (IIBa.9A; 2/1)

Excluding Schoepfia (Q46.278).

Q46.274. Opiliaceae 山柚科 (三IIBa.10A)

Q46.275. Balanophoraceae 蛇苽科 (三IIBa.12)

Q46.276. Santalaceae 檀香科 (IIBa.10; 1/3)

Thesium (百荵草屬); including IIBa.11 Loranthaceae pro parte (Korthalsella 檜葉寄生屬 and Viscum 槲寄生屬).

Q46.278. Schoepfiaceae 青皮木科 (1)

Schoepfia 青皮木屬 (IIBa.9A Olacaceae)

Q46.279. Loranthaceae 桑寄生科 (IIBa.11; 4/2) Loranthus 桑寄生屬 and Taxillus 鈍果桑寄生 屬; excluding Q46.276 Santalaceae pro parte (Korthalsella and Viscum).

R. Caryophyllanae 石竹超目

R47. Caryophyllales 石竹目 R47.281. Tamaricaceae 檉柳科 (1)

Tamarix 檉柳屬 (Wu et al. 2010). R47.282. Plumbaginaceae 藍雪科 (三IIBa.113)

R47.283. Polygonaceae 苶科 ( $\equiv$ IIBa.13)

R47.284. Droseraceae 茅膏菜科 (三IIBa.47)

R47.295. Caryophyllaceae 石竹科 (三IIBa.19)

R47.297. Amaranthaceae 莧科 (IIBa.21; 9/14)

Adding Digera 瘤果莧 (Wang and Chen 2013) and Pupalia 鉤牛膝屬 (Wu et al. 2010); including IIBa.20 Chenopodiaceae (Atriplex 濱㢣屬, Chenopodium 菉屬, and Suaeda 鹼蓬屬).
R47.304. Aizoaceae 番杏科 ( $\equiv$ IIBa.17)

R47.305. Phytolaccaceae 商陸科 (IIBa.14; 2/1)

(Wu et al. 2010)

Excluding Rivina (R47.306).

R47.306. Petiveriaceae 蒜香草科 (1)

Rivina 珊瑚珠屬 (IIBa.14 Phytolaccaceae; Wu et al. 2010).

R47.308. Nyctaginaceae 紫茉莉科 (三IIBa.15)

R47.309. Molluginaceae 粟米草科 (三IIBa.16)

*R47.311. Didiereaceae 刺戟木 (龍樹) 科

R47.312. Basellaceae 落葵科 (三IIBa.18A)

R47.314. Talinaceae 土人參科 (1)

Talinum 土人參屬 (IIBa.18 Portulacaceae).

R47.315. Portulacaceae 馬齒莧科 (IIBa.18;2/1)

Excluding Talinum (R47.314).

R47.317. Cactaceae 仙人掌科 (4)

Cereus 六角柱屬, Epiphyllum 昙花屬, Hylocereus 量天尺屬, and Opuntia 仙人掌屬 (Wu et al. 2010).

\section{S. Asteranae 菊超目}

S48. Cornales 山荣英目

"S48.318. Nyssaceae 珙桐科

S48.320. Hydrangeaceae 八仙花 (繡球) 科 (2)

IIBa.53 Saxifragaceae pro parte [Deutzia 溲 疏屬and Hydrangea (including Cardiandra, Pileostegia, and Schizophragma) 八仙花屬 (De Smet et al. 2015)].

S48.324. Cornaceae 山荣英科 (IIBa.105: 4/2)

Cornus (including Benthamidia and Swida) 山 荣英屬; including IIBa.104 Alangiaceae (Alangium 八角楓屬); excluding Aucuba (S52.351) and Helwingia (S58.391).

S49. Ericales 杜鵑花目

S49.325. Balsaminaceae 鳳仙花科 (三IIBa.73)

"S49.329. Polemoniaceae 花荢 (花蔥) 科

S49.330. Lecythidaceae 玉荵科 (三IIBa.97)

S49.332. Pentaphylacaceae 五列木科 (5)

IIBa.45 Theaceae pro parte (Adinandra 楊桐 屬, Anneslea 茶梨屬, Cleyera 紅淡比屬, Eurya 柃木屬, and Ternstroemia 厚皮香屬).

S49.333. Sapotaceae 山欖科 (三IIBa.114)

S49.334. Ebenaceae 柿樹科 (三IIBa.115)

S49.335. Primulaceae 報春花科 (IIBa.112; 5/8)

Androsace 點地梅屬, Lysimachia (including Anagalis; Manns and Anderberg 2009) 珍珠 菜屬, Primula 櫻草屬, and Stimpsonia 施丁草 屬; including IIBa.111 Myrsinaceae (Ardisia 紫 金牛屬, Embelia 藤木檞屬, Maesa 山桂花屬, and Myrsine 竹杞屬).

S49.336. Theaceae 茶科 (IIBa.45; 9/4) 
Camellia 山茶屬, Gordonia 大頭茶屬, Pyrenaria 烏皮茶屬, and Schima 木荷屬; excluding S49.332 Pentaphylacaceae (Adinandra, Anneslea, Cleyera, Eurya, and Ternstroemia)

S49.337. Symplocaceae 灰木科 (三IIBa.117)

S49.338. Diapensiaceae 岩梅科 (三IIBa.108)

S49.339. Styracaceae 安息香科 (三IIBa.116)

S49.342. Actinidiaceae 晒猴桃科 (三IIBa.44)

S49.345. Mitrastemonaceae 奴草 (帽荵草) 科 (1)

Mitrastemon 奴草屬 (IIBa.43: Rafflesiaceae).

S49.346. Ericaceae 杜鵑花科 (IIBa.110; 6/11)

Including IIBa.109 Pyrolaceae (Cheilotheca 水 晶蘭屬, Chimaphila 愛冬葉屬, Moneses 單花 鹿蹄草屬, Monotropa 錫杖花屬, and Pyrola 鹿蹄草屬)。

S50. Icacinales 茶茱英目

S50.348. Icacinaceae 茶茱英科 (IIBa.78; 3/1)

Nothapodytes 鷹紫花樹屬; excluding Gomphandra (S58.388) and Gonocaryum (S58.389).

S51. Metteniusales 水蟌花目

S52. Garryales 絲縜花目

"S52.350. Eucommiaceae 杜仲科

S52.351. Garryaceae 絲纓花科 (1)

Aucuba 桃葉珊瑚屬 (IIBa.105 Cornaceae).

S53. Gentianales 龍膽目

S53.352. Rubiaceae 茜草科 (IIBa.123; 38/46)

Argostemma 水冠草屬, Canthium 朴萊木屬, Cephalanthus 風箱樹屬, Coptosapelta 漂簐 藤屬, Damnacanthus 伏牛花屬, Dentella 小 牙草屬, Diodia 鈕扣草屬, Galium 豬殊殊屬, Gardenia 黃槴屬, Geophila 苍花蔓屬, Guettarda 葛塔德木屬, Hedyotis 耳草屬, Ixora 仙 丹花屬, Knoxia 諾氏草屬, Lasianthus 雞屎 樹屬, Litosanthes 壼冠木屬, Mitchella 蔓虎 刺屬, Mitracarpus 蓋裂果屬 (Ling and Chen 2013), Morinda 羊角藤屬, Mussaenda 玉葉金 花屬, Neanotis 新耳草屬, Neonauclea 欖仁舅 屬, Nertera 深柱夢草屬, Oldenlandiopsis微耳 草屬 (Jung et al. 2011), Ophiorrhiza 蛇根草屬 (including Hayataella; Nakamura et al. 2006), Paederia 雞屎藤屬, Pavetta 茜木屬, Psychotria 九節木屬, Randia 茜草樹屬, Richardia 擬鴨舌癀屬, Rubia 茜草屬, Serissa 滿天星屬 (Wu et al. 2010), Sherardia 雪亞迪草屬 (Wu et al. 2010), Sinoadina 水冬瓜屬, Spermacoce 擬鴨舌癀舅屬 (includng Hemidiodia in Wu et al. 2010), Tarenna 玉心花屬, Timonius 貝 木屬, Tricalysia 狗骨仔屬, Uncaria 鉤藤屬, and Wendlandia 水錦樹屬; including IIBa.103
Theligonaceae (Theligonum 纖花草屬). Based on Neupane et al. (2015), the genera Dimetia 涼喉茶屬 (新擬), Exallage 金毛耳草屬 (新擬), Leptopetalum 脈耳草屬 (新擬), Oldenlandia 龍吐珠屬 (新擬), and Scleromitrion 蛇舌草屬 are segregated from the Taiwanese species of Hedyotis (Hsu and Chen 2017).

S53.353. Gentianaceae 龍膽科 (IIBa.120; 7/7) Including Fagraea 灰莉屬 (IIBa.119 Loganiaceae); excluding Nymphoides (S59.400).

S53.354. Loganiaceae 馬錢科 (IIBa.119; 6/4) Excluding Fagraea (S53.353) and Buddleja (S57.371).

S53.356. Apocynaceae 夾竹桃科 (IIBa.121; 10/24) Alyxia 念珠藤屬, Anodendron 錦蘭屬, Cerbera海檬果屬, Holarrhena 止瀉木屬, Melodinus 山橙屬, Parsonsia 爬森藤屬, Rauvolfia 蘿芙木屬, Tabernaemontana 馬蹄花 屬, Trachelospermum絡石屬, Urceola (including Ecdysanthera) 水壼藤屬; adding Alstonia 黑板樹屬 (Wu et al. 2010) and Catharanthus 長春花屬 (Vinca in Wu et al. 2010); including IIBa.122 Asclepiadaceae (Asclepias 尖尾 鳳屬, Cryptolepis 隱鱗藤屬, Cynanchum 牛皮 消屬, Dischidia 風不動屬, Dregea 華他卡藤 屬, Gymnema 武靴藤屬, Heterostemma 布朗 藤屬, Hoya 速蘭屬, Jasminanthes 舌瓣花屬, Marsdenia 牛彌菜屬, Telosma 夜香花屬, and Tylophora 鷗蔓屬).

S54. Boraginales 紫草目 (sensu Luebert et al. 2016) S54.357. Boraginaceae 紫草科 (IIBa.126; 12/8) Bothriospermum 細纍子草屬, Cynoglossum 琉璃草屬, Lithospermum 紫草屬, Thyrocarpus 盾果草屬, Trichodesma 碧果草屬, and Trigonotis 附地草屬; adding Myosotis 勿忘草 屬 (Wu et al. 2010) and Symphytum 聚合草 屬 (Wu et al. 2010); excluding S54.357B Coldeniaceae (Coldenia), S54.357C Cordiaceae (Cordia), S54.357D Ehretiaceae (Carmona and Ehretia), and S54.357E Heliotropiaceae (Heliotropium and Tournefortia).

S54.357B. Coldeniaceae 生果草科 (1) Coldenia 生果草屬 (IIBa.126 Boraginaceae).

S54.357C. Cordiaceae 破布子科 (1) Cordia 破布子屬 (IIBa.126 Boraginaceae).

S54.357D. Ehretiaceae 厚殼樹科 (1) Ehretia (including Carmona) 厚殼樹屬 (IIBa.126 Boraginaceae).

S54.357E. Heliotropiaceae 天芹菜科 (1) Heliotropium (including Tournefortia) 天芹菜 屬 (IIBa.126 Boraginaceae). 
S55. Vahliales 黃漆姑目

S56. Solanales 茄目

S56.359. Convolvulaceae 旋花科 (三IIBa.124)

S56.360. Solanaceae 茄科 (三 IIBa.130)

S56.362. Sphenocleaceae 密穗桔梗 (尖瓣花) 科 ( $\equiv$ IIBa.142A)

S56.363. Hydroleaceae 田基麻科 (1)

Hydrolea 探芹草屬 (IIBa.125 Hydrophyllaceae)

S57. Lamiales 唇形目

S57.366. Oleaceae 木犀科 (三IIBa.118)

S57.369. Gesneriaceae 苦苍苔科 (三IIBa.134)

S57.370. Plantaginaceae 車前科 (IIBa.138; 1/16)

Plantago 車前屬; including IIBa.128 Callitrichaceae (Callitriche 水馬齒屬) and IIBa.131 Scrophulariaceae pro parte [Antirrhinum 金魚 草屬 (Chen and Wang 2014), Bacopa 過長沙 屬, Deinostema 澤番椒屬, Digitalis 毛地黃屬, Dopatrium 虹眼草屬, Ellisiophyllum 海螺菊 屬, Hemiphragma 腰只花屬, Limnophila 石龍 尾屬, Mecardonia 過長沙舅屬, Microcarpaea 微果草屬, Scoparia 野甘草屬, Stemodia (變 生花屬; Liang et al. 2011), Veronica 婆婆納屬, and Veronicastrum 腹水草屬].

S57.371. Scrophulariaceae 玄參科 (IIBa.131; 26/3) Scrophularia (玄參屬); including Buddleja 揚波屬 (IIBa.119 Loganiaceae) and IIBa.137 Myoporaceae (Myoporum 苦藍盤屬); excluding S57.370 Plantaginaceae pro parte (Bacopa, Deinostema, Digitalis, Dopatrium, Ellisiophyllum, Hemiphragma, Limnophila, Mecardonia, Microcarpaea, Scoparia, Veronica, and Veronicastrum), S57.373 Linderniaceae (Legazpia, Lindernia, and Torenia), S57.384 Mazaceae (Mazus), S57.385 Phrymaceae (Mimulus), S57.386 Paulowniaceae (Paulownia), and S57.387 Orobanchaceae pro parte (Alectra, Centranthera, Euphrasia, Pedicularis, Phtheirospermum, Siphonostegia, and Striga).

S57.373. Linderniaceae 母草科 (4)

IIBa.131 Scrophulariaceae pro parte [Legazpia 三翅䓵屬, Lindernia 母草屬, Micranthemum 珍珠草屬 (Hsu et al. 2016a), and Torenia 倒地 蜈蚣屬]

"S57.376. Pedaliaceae 胡麻 (芝麻) 科

S57.377. Acanthaceae 爵床科 (IIBa.133; 15/20)

Adding Asystasia 十萬錯屬 (Wu et al. 2010), Nelsonia 瘤子草屬 (Wang et al. 2016), and Thunbergia 鄧伯花屬 (Wu et al. 2010); including Avicennia 海茄苳屬 (IIBa.127 Verbenaceae).
S57.378. Bignoniaceae 紫葳科 (三IIBa.132)

S57.379. Lentibulariaceae 狸藻科 (三IIBa.136)

S57.382. Verbenaceae 馬鞭草科 (IIBa.127; 11/5)

Lantana 馬縜丹屬, Phyla 鴨舌癀屬, Stachytarpheta 木馬鞭屬, Verbena 馬鞭草屬; adding Duranta 假連趐屬 (Wu et al. 2010); excluding Avicennia (S57.377) and S57.383 Lamiaceae pro parte (Callicarpa, Caryopteris, Clerodendrum, Premna, Sphenodesme, and Vitex).

S57.383. Lamiaceae (三 Labiatae) 唇形科 (IIBa.129; 36/43)

Including IIBa.127 Verbenaceae pro parte [Callicarpa 紫珠屬, Caryopteris 蓜屬, Clerodendrum 海州常山屬, Premna 魚臭木屬, Sphenodesme 楔翅藤屬, Tectona 柚木屬 (Wu et al. 2010), and Vitex 牡荊屬].

S57.384. Mazaceae 通泉草科 (1)

Mazus 通泉草屬 (IIBa.131 Scrophulariaceae)

S57.385. Phrymaceae 蠅毒草 (透骨草) 科 (3)

Erythranthe (三 Mimulus; Barker et al. 2012) 溝

酸漿屬 (IIBa.131 Scrophulariaceae), Peplidium

溝馬齒屬 (Hsu et al. 2016b), and Phryma 蠅毒 草屬 (Jung et al. 2005).

S57.386. Paulowniaceae 泡桐科 (1)

Paulownia 泡桐屬 (IIBa.131 Scrophulariaceae). S57.387. Orobanchaceae 列當科 (IIBa.135; 4/14)

Aeginetia 野菰屬, Boschniakia 草蓯容屬, Christisonia 假野菰屬, Orobanche 列當屬; adding Phacellanthus 黃筒花屬 (Chung et al. 2010b); including IIBa.131 Scrophulariaceae pro parte [Alectra 黑蒴屬, Centranthera 距芯 花屬, Euphrasia 碎雪草屬, Lathraea 齒鱗草 屬 (Chung et al. 2010a), Melampyrum山羅花 屬 (Chen and Wang 2009), Pedicularis 馬先蒿 屬, Phtheirospermum 松蒿屬, Siphonostegia 陰 行草屬, and Striga 獨腳金屬].

S58. Aquifoliales 冬青目

S58.388. Stemonuraceae 金檀木 (粗絲木) 科 (1)

Gomphandra 毛芯木屬 (IIBa.78 Icacinaceae)

S58.389. Cardiopteridaceae 心翼果科 (1)

Gonocaryum 瓊欖屬 (IIBa.78 Icacinaceae)

S58.391. Helwingiaceae 青萊葉科 (1)

Helwingia 青萊葉屬 (IIBa.105 Cornaceae)

S58.392. Aquifoliaceae 冬青科 (三 IIBa.74)

S59. Asterales 菊目

S59.394. Campanulaceae 桔梗科 (三 IIBa.142)

S59.400. Menyanthaceae 睡菜科 (1)

Nymphoides 苦菜屬 (IIBa.120 Gentianaceae) S59.401. Goodeniaceae 草海桐科 (三IIBa.143) S59.403. Asteraceae (三 Compositae) 菊科 (三 IIBa.144) 
S60. Escalloniales 南鼠刺 (吊片果) 目

S61. Bruniales 絨球花目

S62. Paracryphiales 盔被花 (盔瓣花) 目

S63. Dipsacales 川續斷目

S63.408. Adoxaceae 五福花科 (2)

IIBa.139 Caprifoliaceae pro parte (Sambucus 接骨木屬 and Viburnum 萊蒾屬).

S63.409. Caprifoliaceae 忍冬科 (IIBa.139; 4/6)

Abelia 六道木屬 and Lonicera 忍冬屬; including IIBa.141 Dipsacaceae (Scabiosa 山蘿葍屬) and IIBa.140 Valerianaceae (Patrinia 敗醬屬, Triplostegia 雙參屬, and Valeriana 續草屬); excluding S63.408 Adoxaceae (Sambucus and Viburnum).

S64. Apiales 繖形目

S64.413. Pittosporaceae海桐科 (三 IIBa.54)

S64.414. Araliaceae五加科 (IIBa.106; 10/11) Including Hydrocotyle 天胡荽屬 (IIBa.107 Umbelliferae).

S64.416. Apiaceae (三 Umbelliferae) 繖形科 (IIBa.107; 18/18)

Adding Foeniculum 茴香屬 (Wu et al. 2010); excluding Hydrocotyle (S64.414).

\section{Additional file}

Additional file 1: Appendix S1. List of seed plant genera of Taiwan and their familial classification under the Phylogenetic Classification of Seed Plants of Taiwan

\section{Abbreviations}

APG: Angiosperm Phylogeny Group; FOT: Flora of Taiwan; TaiBIF: Taiwan Biodiversity Information Facility; TaiCOL: Catalogue of Life in Taiwan; TaiEOL: Taiwan Encyclopedia of Life; TaiBOL: Cryobanking Program for Wildlife Genetic Material in Taiwan; TELDAP: Taiwan e-Learning and Digital Archives Programs; GBIF: Global Biodiversity Information Facility; TaiBNET: Taiwan Biodiversity National Information Network.

\section{Authors' contributions}

C-TL and K-FC conceived the idea, designed the project, and wrote the manuscript. C-TL conducted data cleaning and data analysis. Both authors read and approved the final manuscript.

\section{Author details}

${ }^{1}$ Department of Biological Resources, National Chiayi University, Chiayi 60004, Taiwan. ${ }^{2}$ Research Museum and Herbarium (HAST), Biodiversity Research Center, Academia Sinica, Taipei 11529, Taiwan.

\section{Acknowledgements}

This work is supported by Forestry Bureau, Council of Agriculture [106AS-11.9.6-FB-e2 (to C-TL), 106-08.1-SB-17(3) (to K-FC), and 106AS-11.8.1FB-e4 (to K-FC)] and Ministry of Science and Technology (MOST105-2621-B001-002-MY3) (to K-FC).

\section{Competing interests}

The authors declare that they have no competing interests.

Availability of data and materials

Not applicable.

\section{Consent for publication}

Not applicable.

\section{Ethics approval and consent to participate}

Not applicable.

\section{Funding}

Supported by Forestry Bureau, Council of Agriculture [106AS-11.9.6-FB-e2 (to C-TL), 106-08.1-SB-17(3) (to K-FC), and 106AS-11.8.1-FB-e4 (to K-FC)] and Ministry of Science and Technology (MOST105-2621-B001-002-MY3) (to K-FC).

\section{Publisher's Note}

Springer Nature remains neutral with regard to jurisdictional claims in published maps and institutional affiliations.

Received: 6 September 2017 Accepted: 10 November 2017 Published online: 21 November 2017

\section{References}

Barker WR, Nesom GL, Beardsley PM, Fraga NS (2012) A taxonomic conspectus of Phrymaceae: a narrowed circumscription for Mimulus, new and resurrected genera, and new names and combinations. Phytoneuron 39:1-60

Boufford DE, Ohashi H, Huang T-C, Hsieh C-F, Tsai J-L, Yang K-C, Peng C-I, Kuoh C-S, Hsiao A (2003) A checklist of the vascular plants of Taiwan. In: Editorial Committee of the Flora of Taiwan (ed) Flora of Taiwan, vol 6, 2nd edn. Department of Botany, National Taiwan University, Taipei, pp 15-139

Chao C-T, Tseng Y-H, Tseng H-Y (2013) Heteropolygonatum altelobatum (Asparagaceae), comb. nova. Ann Bot Fennici 50:91-94. https://doi. org/10.5735/085.050.0117

Chao C-T, Chen P-H, Tseng H-Y (2017) Linum usitatissimum L. (Linaceae), a newly naturalized species in Taiwan. Quart J For Res 39:107-111

Chapman AD (2005) Principles of data quality, version 10. Global Biodiversity Information Facility, Copenhagen

Chase MW, Reveal JL (2009) A phylogenetic classification of the land plants to accompany APG III. Bot J Lin Soc 161:122-127. https://doi. org/10.1111/j.1095-8339.2009.00996.x

Chen C-H, Wang C-M (2009) Melampyrum roseum Maxim. (Scrophulariaceae), a newly recorded genus and species in Taiwan. Taiwania 54:183-186

Chen C-H, Wang C-M (2014) Antirrhinum orontium L. (Scrophulariacaee), a newly naturalized genus and species in Taiwan. Coll Res 27:71-75

Christenhusz MJM, Reveal JL, Farjon A, Garden MF, Mill RR, Chase MW (2011) A new classification and linear sequence of extant gymnosperms. Phytotaxa 19:55-70. https://doi.org/10.11646/phytotaxa.19.1.3

Chung S-W, Hsu T-C, Jung M-J, Hsiao S-C, Fang W-U (2010a) Lathraea purpurea (Scrophulariaceae): a new generic record in Taiwan. Taiwan J For Sci 25:265-269

Chung S-W, Hsu T-C, Peng C-I (2010b) Phacellanthus (Orobanchaceae), a newly recorded genus in Taiwan. Bot Stud 51:531-536

Cronquist A (1968) The evolution and classification of flowering plants. Houghton Mifflin, Boston

De Smet Y, Granados Mendoza C, Wanke S, Goetghebeur P, Samain M-S (2015) Molecular phylogenetics and new (infra) generic classification to alleviate polyphyly in tribe Hydrangeeae (Cornales: Hydrangeaceae). Taxon 64:741-753. https://doi.org/10.12705/644.6

Herbert J (2005) New combinations and a new species in Morella (Myricaceae). Novon 15:293-295

Hsieh C-F (2003) Composition, endemism and phytogeographical affinities of the Taiwan flora. In: Editorial Committee of the Flora of Taiwan (ed) Flora of Taiwan, vol 6, 2nd edn. Department of Botany, National Taiwan University, Taipei, pp 1-14 
Hsu TC, Chen ZH (2017) Scleromitrion sirayanum (Rubiaceae: Spermacoceae), a new species of the Hedyotis-Oldenlandia complex in Taiwan. Taiwania 62:151-156. https://doi.org/10.6165/tai.2017.62.151

Hsu T-W, Chiang T-Y, Chiang Y-C (2016b) Peplidium maritimum (L.f.) Asch. (Phrymaceae), a new record in the Flora of Taiwan. Taiwan J Biodivers 18:253-258

Hsu TW, Chiang TY, Huang CC (2016a) Two newly naturalized species in Taiwan, Rnellia squarrosa (Fenzl) Schaffnit and Micranthemum micrathemoides (Nutt.) Wettst. Taiwan J Biodivers 18:123-130

Hsu T-W, Kono Y, Chiang T-Y, Peng C-I (2011) Ypsilandra (Melanthiaceae: Liliaceae sensu lato), a new generic record for Taiwan. Bot Stud 52:99-104

Huang TC, Huang SF, Hsieh TH (1994) The flora of Tungshatao (Pratas Island). Taiwania 39:27-53. https://doi.org/10.6165/tai.1994.39.27

Huang T-C (1994) Preface of Volumen One. In: Editorial Committee of the Flora of Taiwan (ed) Flora of Taiwan, vol 1, 2nd edn. Department of Botany, National Taiwan University, Taipei, $\mathrm{pp} v$-vi

Huguet V, Gouy M, Normand P, Zimpfer JF, Fernandez MP (2005) Molecular phylogeny of Myricaceae-a reexamination of host-symbiont specificity. Mol Phylogenet Evol 34:557-568. https://doi.org/10.1016/j. ympev.2004.11.018

Jung M-J, Ku S-M, Peng C-I (2011) Oldenlandiopsis Terell \& W H. Lewis (Rubiaceae), a newly recorded genus in Taiwan. Taiwania 56:58-61

Jung M-J, Liao G-I, Kuoh C-S (2005) Phryma leptostachya (Phrymaceae), a new family record in Taiwan. Bot Bull Acad Sinica 46:239-244

Kanis A, Crisp MD, Orchard AE (1999) Classification, phylogeny and the flora of Australia. In: Orchard AE (ed) Flora of Australia, vol 1 Introduction. CSIRO Publishing, Canberra, pp 125-147

Ko CJ (2004) A taxonomic and distributional study of seagrasses in Taiwan. Master thesis, National Sun Yat-sen University

Les DH, Crawford DJ (1999) Landoltia (Lemnaceae), a new genus of duckweeds. Novon 9:530-533

Li C-Y, Wang C-M (2012) Anoda cristata (L.) Schltdl. (Malvaceae), a newly naturalized plant in Taiwan. Quart J For Res 34:263-268

Liang Y-S, Jung M-J, Wu S-C, Kao Y-C, Wang J-C (2011) Stemodia L. (Scrophulariaceae), a newly naturalized genus in Taiwan. Taiwania 56:62-65

Lin HJ, Hsieh LY, Liu PJ (2005) Seagrasses of Tongsha Island, with descriptions of four new records to Taiwan. Bot Bull Acad Sin 46:163-168

Lindqvist C, De Laet J, Haynes RR, Aagesen L, Keener BR, Albert VA (2006) Molecular phylogenetics of an aquatic plant lineage, Potamogetonaceae. Cladistics 22:568-588. https://doi.org/10.1111/j.1096-0031.2006.00124.x

Ling C-J, Chen C-F (2013) Mitracarpus hirtus (L.) DC. (Rubiaceae), a newly naturalized plant in Taiwan. Taiwan J Biodivers 15:155-161

Liu B, Ye J, Liu S, Wang Y, Yang Y, Lai Y, Zeng G, Lin Q (2015) Families and genera of Chinese angiosperms: a synopsis classification based on APG III. Biodivers Sci 23:225-231. https://doi.org/10.17520/biods.2015052

Lu Y, Ran J-H, Guo D-M, Yang Z-Y, Wang X-Q (2014) Phylogeny and divergence times of gymnosperms inferred from single-copy nuclear genes. PLoS ONE 9:e107679. https://doi.org/10.1371/journal.pone.0107679

Luebert F, Cecchi L, Frohlich MW, Gottschling M, Guilliams CM, HasenstabLehman KE, Hilger HH, Miller JS, Mittelbach M, Nazaire M, Nepi M, Nocentini D, Ober D, Olmstead RG, Selvi F, Simpson MG, Sutory K, Valdes B, Walden GK, Weigend M (2016) Familial classification of the Boraginales. Taxon 65:502-522. https://doi.org/10.12705/653.5

Maletic Jl, Marcus A (2000) Data cleansing: beyond integrity analysis proceedings of the 2000 conference on information quality, 200-209. MIT, Cambridge

Manns U, Anderberg AA (2009) New combinations and names in Lysimachia (Myrsinaceae) for species of Anagallis, Pelletiera and Trientalis. Willdenowia 39:49-54. https://doi.org/10.3372/wi.39.39103

Mayr E (1981) Biological classification: toward a synthesis of opposing methologies. Science 214:510-516

Nakamura K, Chung S-W, Kokubugata G, Denda T, Yokota M (2006) Phylogenetic systematics of the monotypic genus Hayataella (Rubiaceae) endemic to Taiwan. J Plant Res 119:657-661. https://doi.org/10.1007/ s10265-006-0017-4
Neupane S, Dessein S, Wikström N, Lewis PO, Long C, Bremer B, Motley TJ (2015) The Hedyotis-Oldenlandia complex (Rubiaceae: Spermacoceae) in Asia and the Pacific: phylogeny revisited with new generic delimitations. Taxon 64:299-322. https://doi.org/10.12705/642.8

Patterson DJ, Cooper J, Kirk PM, Pyle RL, Remsen DP (2010) Names are key to the big new biology. Trends Ecol Evol 25:686-691. https://doi. org/10.1016/.tree.2010.09.004

Patterson DJ, EgloffW, Agosti D, Eades D, Franz N, Hagedorn G, Rees JA, Remsen DP (2014) Scientific names of organisms: attribution, rights, and licensing. BMC Res Notes 7:e79. https://doi.org/10.1 186/1756-0500-7-79

Reveal JL (1993) Flowering plant families: an overview. In: Flora of North America Editorial Committee (ed) Flora of North America North of Mexico, vol 1. Oxford University Press, New York, pp 294-330

Ruggiero MA, Gordon DP, Orrell TM, Bailly N, Bourgoin T, Brusca RC, CavalierSmith T, Guiry MD, Kirk PM (2015) A higher level classification of all living organisms. PLoS ONE 10:e0119248. https://doi.org/10.1371/journal. pone. 0119248

Shao K-T, Lai K-C, Lin Y-C, Chen L-S, Li H-Y, Hsu C-H, Lee H, Hsu H-W, Mai G-S (2013) Experience and strategy of biodiversity data integration in Taiwan. Data Sci J 12:WDS61-WDS69. https://doi.org/10.2481/dsj.wds-008

Shao K-T, Peng C-I, Wu W-J (eds) (2008) Taiwan species diversity II. Species checklist. Forestry Bureau, Council of Agriculture, Executive Yuan, Taipei

The Angiosperm Phylogeny Group (1998) An ordinal classification for the families of flowering plants. Ann Mo Bot Gard 85:531-553. https://doi. org/10.2307/2992015

The Angiosperm Phylogeny Group (2003) An update of the Angiosperm Phylogeny Group classification for the orders and families of flowering plants: APG II. Bot J Linn Soc 141:399-436. https://doi. org/10.1046/j.1095-8339.2003.t01-1-00158.x

The Angiosperm Phylogeny Group (2009) An update of the Angiosperm Phylogeny Group classification for the orders and families of flowering plants: APG III. Bot J Linn Soc 161:105-121. https://doi. org/10.1111/j.1095-8339.2009.01002x

The Angiosperm Phylogeny Group (2016) An update of the Angiosperm Phylogeny Group classification for the orders and families of flowering plants: APG IV. Bot J Linn Soc 181:1-20. https://doi.org/10.1111/boj.12385

Thulin M, Moore AJ, El-Seedi H, Larsson A, Christin PA, Edwards EJ (2016) Phylogeny and generic delimitation in Molluginaceae, new pigment data in Caryophyllales, and the new family Corbichoniaceae. Taxon 65:775-793. https://doi.org/10.12705/654.6

Trias-Blasi A, Baker WJ, Haigh AL, Simpson DA, Weber O, Wilkin P (2015) A genus-level phylogenetic linear sequence of monocots. Taxon 64:552581. https://doi.org/10.12705/643.9

Wang C-M, Chang K-C, Chen C-H (2016) Two newly naturalized plant species in Taiwan: Fumaria parviflora Lam. and Nelsonia canescens (Lam.) Spreng. Taiwan J For Sci 31:135-141

Wang C-M, Chen C-H (2013) Digera muricata (L.) Mart. (Amaranthaceae), a newly naturalized genus and species in Taiwan. Taiwania 58:194-198. https://doi.org/10.6165/tai.2013.58.194

Wang X-Q, Ran J-H (2014) Evolution and biogeography of gymnosperms. Mol Phylogenet Evol 75:24-40. https://doi.org/10.1016/.ympev.2014.02.005

Wearn JA, Chase MW, Mabberley DJ, Couch C (2013) Utilizing a phylogenetic plant classification for systematic arrangements in botanic gardens and herbaria. Bot J Linn Soc 172:127-141. https://doi.org/10.1111/boj.12031

Wu Y-H, Chao C-T, Tseng Y-H (2015) Eurya lui (Pentaphylacaceae). Quart J For Res 37:219-228

Wu S-H, Yang T-YA, Teng Y-C, Chang C-Y, Yang K-C, Hsieh C-F (2010) Insights of the latest naturalized flora of Taiwan: change in the past 8 years. Taiwania 55:139-159

Yamashita J, Tamura MN (2004) Phylogenetic analyses and chromosome evolution in Convallarieae (Ruscaceae sensu lato), with some taxonomic treatments. J Plant Res 117:363-370. https://doi.org/10.1007/ s10265-004-0169-Z

Yang Y-P, Yen S-H, Lin C-K (2001) Illustrated guide to aquatic plants of Taiwan. Council of Agriculture, The Execuative Yuan of Taiwan, Taipei 